\title{
BMJ Open Preferences for healthcare services among hypertension patients in China: a discrete choice experiment
}

\author{
Xiaolan Yu, ${ }^{1}$ Haini Bao, ${ }^{1}$ Jianwei Shi (D) , ${ }^{2}$ Xiaoyu Yuan, ${ }^{3}$ Liangliang Qian, ${ }^{4}$ \\ Zhe Feng, ${ }^{1}$ Jinsong Geng (i) ${ }^{1}$
}

To cite: Yu X, Bao H, Shi J, et al. Preferences for healthcare services among hypertension patients in China: a discrete choice experiment. BMJ Open 2021;11:e053270. doi:10.1136/ bmjopen-2021-053270

- Prepublication history and additional supplemental material for this paper are available online. To view these files, please visit the journal online (http://dx.doi.org/10.1136/ bmjopen-2021-053270)

$\mathrm{XY}$ and $\mathrm{HB}$ contributed equally.

$\mathrm{XY}$ and $\mathrm{HB}$ are joint first authors.

Received 08 May 2021

Accepted 16 November 2021

\section{Check for updates}

(c) Author(s) (or their employer(s)) 2021. Re-use permitted under CC BY-NC. No commercial re-use. See rights and permissions. Published by BMJ.

${ }^{1}$ Department of Medical Informatics, Nantong University Medical School, Nantong, Jiangsu, China

${ }^{2}$ School of Public Health, Shanghai Jiao Tong University School of Medicine, Shanghai, China

${ }^{3}$ Department of Emergency Medicine, Affiliated Hospital of Nantong University, Nantong, Jiangsu, China

${ }^{4}$ Department of Family Health Services, Pujiang Community Health Service Center, Shanghai, China

Correspondence to Professor Jinsong Geng; gjs@ntu.edu.cn

\section{ABSTRACT}

Objectives Our study aimed to support evidence-informed policy-making on patient-centred care by investigating preferences for healthcare services among hypertension patients

Design We identified six attributes of healthcare services for a discrete choice experiment (DCE), and applied Bayesian-efficient design with blocking techniques to generate choice sets. After conducting the DCE, we used a mixed logit regression model to investigate patients' preferences for each attribute and analysed the heterogeneities in preferences. Estimates of willingness to pay were derived from regression coefficients.

Setting The DCE was conducted in Jiangsu province and Shanghai municipality in China.

Participants Patients aged 18 years or older with a history of hypertension for at least 2 years and who took medications regularly were recruited.

Results Patients highly valued healthcare services that produced good treatment effects $(\beta=4.502, p<0.001)$, followed by travel time to healthcare facilities within 1 hour $(\beta=1.285, p<0.001)$, and the effective physician-patient communication ( $\beta=0.771, p<0.001$ ). Continuity of care and minimal waiting time were also positive predictors $(p<0.001)$. However, the out-of-pocket cost was a negative predictor of patients' choice $(\beta=-0.168, p<0.001)$. Older adults, patients with good health-related quality of life, had comorbidities, and who were likely to visit secondary and tertiary hospitals cared more about favourable effects $(p<0.05)$. Patients were willing to pay $¥ 2489(95 \% \mathrm{Cl}$ $¥ 2013$ to $¥ 2965$ ) as long as the clinical benefits gained were substantial.

Conclusions Our findings highlight the importance of effective, convenient, efficient, coordinated and patientcentred care for chronic diseases like hypertension. Policymakers and healthcare providers are suggested to work on aligning the service provision with patients' preferences.

\section{INTRODUCTION}

Hypertension, also known as high blood pressure, is a condition in which the blood vessels have raised pressure persistently. Hypertension can damage the brain, heart, kidney and arterial blood vessels. It is ranked as the leading cause of premature death and the most important modifiable risk factor for cardiovascular disease. ${ }^{1}$ The prevalence

\section{Strengths and limitations of this study}

The discrete choice experiment is a rigorous method that enables us to measure preferences for healthcare services among hypertension patients.

- Bayesian-efficient design with a blocking technique was applied to improve statistical efficiency as well as response efficiency.

- Comorbidities, past healthcare experience and health-related quality of life were used as variables to observe preference heterogeneity and address evidence gaps.

- While this study explored the preferences among hypertension patients, future studies need to examine other types of chronic diseases.

of hypertension is high and continues to be rising in China in recent years. Among Chinese adults aged over 15 years, $18.14 \%$ have hypertension. ${ }^{2}$ Despite huge efforts, the awareness, treatment and control rate of hypertension remained extremely low, which were associated with substantial unnecessary disease burden and significant excess mortality. ${ }^{3-5}$ Moreover, many hypertension patients have multiple comorbidities, which is associated with increased utilisation of healthcare services and great financial burden to individuals and the health system. ${ }^{67}$

To optimise the allocation of healthcare resources and reach the goal of delivering high-standard healthcare services, since 2009, the Chinese government has vigorously promoted the implementation of the hierarchical medical system. Primary healthcare facilities like community health service centres are expected to offer affordable first-contact care, while secondary and tertiary hospitals provide specialist referral services. In the past decade, advances have been made by the Chinese government in achieving universal health coverage and providing financial protection for its 
citizens. ${ }^{8}$ However, primary healthcare was underused, and the referral system was still practised with poor effectiveness. ${ }^{9}$

Patients were more favourable to healthcare services in hospitals than primary healthcare facilities in China. ${ }^{10}$ Individuals with better socioeconomic status and greater healthcare needs seemed to be less likely to use primary healthcare. ${ }^{11}$ As a result, hospitals were overloaded, and the long waiting time became the major source of dissatisfaction. ${ }^{12}$ On the contrary, an integrated delivery system based on primary healthcare is helpful to meet the needs of China's ageing population that are facing an increased chronic disease burden. ${ }^{13}$ Nevertheless, patients' preferences for hospital-based services for first-contact care place a huge obstacle to promoting community-based primary healthcare service. ${ }^{14}$

Understanding patients' preferences are particularly worthwhile when patient decisions are preferencesensitive, like the choice in healthcare services. Eliciting patients' preferences is a key element of patient-centred care. The discrete choice experiment (DCE) is a wellestablished quantitative approach to elicit stated preferences. Despite several DCEs being carried out to investigate patients' preferences for healthcare services, none of them involved patients with hypertension in China, one of the most common types of chronic diseases. $^{15-19}$

Although patient-reported outcomes, such as healthrelated quality of life (HRQoL) are essential measures of health status, whether patients' preferences on healthcare services differ from HRQoL remain unclear. Furthermore, preferences contain a learnt component, and past experience might influence an individual's present choice. ${ }^{2021}$ We remain unclear about whether the healthcare facilities that patients usually visited in the past could have an impact on their current preferences for healthcare services.

Due to the high prevalence, serious complications and heavy burden, hypertension has become an important public health challenge. Effective and efficient healthcare services for hypertension patients are essential to successful disease control. Meanwhile, patients' demand for healthcare services varies according to the severity of the disease. ${ }^{22} 23$ Therefore, we aim to fill the gap by measuring preferences of healthcare services for first-contact care among hypertension patients, thus supporting evidence-informed policy-making to address the problems of inappropriate healthcare service utilisation. Specifically, we conducted a DCE to test the following hypotheses: (1) attributes regarding health benefits are more important than other attributes for patients' preferences of healthcare services for first-contact care; (2) patients' preferences differ by sociodemographic characteristics, feelings of health status (ie, HRQoL), the severity of disease (ie, comorbidities) and the prior experience of healthcare services.
METHODS

\section{Identification of attributes and levels}

Our DCE design, implementation and analysis followed the user guide jointly developed by the World Bank, WHO and the US Agency for International Development. ${ }^{24}$ First, a literature search on February 10th, 2020, was conducted to identify attributes that were used in DCEs regarding preferences of healthcare services among patients with chronic diseases or chronic conditions. Twenty-seven studies were identified, with one $\mathrm{DCE}^{25}$ conducted in the UK aimed to explore patients' preferences for the management of hypertension (online supplementa appendix 1). We found that the most commonly mentioned domains were the service provision capabilities (skills and attitudes of medical staff, accessibility of medicines and medical equipment, clinical benefits, environment, continuity of the care/coordination and continuity), service efficiency (waiting time for the appointment or treatment), affordability (costs or out-of-pocket costs for healthcare services), and convenience (travel time or distance from home to healthcare facilities) (online supplemental appendix 2). While in the DCE for preferences of hypertension patients, ${ }^{25}$ there were four attributes including service provision capabilities (frequency of blood pressure measurement), clinical benefits (reduction in 5-year cardiovascular risk), affordability (at the macro level as measured by the annual cost to National Health Service in the UK), and model of care (as defined by types of personnel who was responsible for disease management).

Second, focus group discussions with physicians and hospital managers were carried out to determine the attributes and levels. According to their suggestions, although the expertise of healthcare professionals was found to be an important attribute for patients' preferences, benefit from healthcare was also indispensable. Effectiveness is one of the important domains in quality assessment measures. ${ }^{2627}$ The effectiveness of healthcare has been considered as the ultimate validator of the quality of care. ${ }^{28}$ Furthermore, improvement in the effectiveness of healthcare service would be helpful to achieve population health improvement and health system sustainability. ${ }^{29}$ Healthcare services that could bring health benefits usually had strong recommendations from experts. ${ }^{30-32}$ As shown in guidelines on management and control of hypertension, getting blood pressure under control and reducing the risk of complications are the goals for hypertension treatment. ${ }^{33} 34$ Therefore, we classified the levels of treatment effects according to the control of blood pressure and complications.

Continuity of care is a necessary part of the framework on integrated people-centred health services proposed by WHO. ${ }^{35}$ Continuity of care was correlated with increased patient satisfaction, and effective physician-patient communication was regarded as a central clinical function. ${ }^{36}$ Traditionally, a continuous caring relationship with the same healthcare professionals was found in previous DCEs. ${ }^{38-42}$ However, for providers in vertically 
Table 1 Attributes and levels of healthcare services in the DCE

\begin{tabular}{llll}
\hline Domains & Attributes & Levels & Variables coding \\
\hline Capabilities & Treatment effects & Good; moderate; poor & Categorical \\
& Physician-patient communication & Good; moderate; poor & Categorical \\
& Continuity of care & Yes; no & Categorical \\
Efficiency & Waiting time & Within 0.5 hour; 2 hours; 4hours or longer & Categorical \\
Affordability & Out-of-pocket costs per visit (if reimbursed) & $¥ 150-¥ 600$ & Continuous \\
Convenience & Travel time & Within 1 hour; 3hours; 6hours or longer & Categorical \\
\hline
\end{tabular}

The average exchange rate of US Dollars to CNY in 2020 was about 6.90 . Therefore, $¥ 150$ was approximately US $\$ 21.7$ and $¥ 600$ was about US\$87.0.

CNY, Chinese Yuan; DCE, discrete choice experiment.

integrated healthcare systems, the contrasting ideal is the delivery of a 'seamless service' ${ }^{43}$ Similarly, multidisciplinary care became an attribute in a DCE that measured preferences for urban integrated primary care among type 2 diabetes patients. ${ }^{44}$ As a result, we defined continuity of care as the healthcare facility operating in a wellfunctioning care delivery system, which could provide coordinated healthcare services for patients. ${ }^{43}$

Attributes and levels of healthcare services that were used in our DCE were shown in table 1. Details of the explanation of attributes and levels were listed in online supplemental appendix 3. Our research objective was to identify the healthcare service attributes and levels that were preferred by hypertension patients, not the grade of hospitals (ie, primary, secondary, tertiary). Hence, the scenarios in our DCE were not restricted to a specific grade of hospitals.

\section{Experimental design and development of the questionnaire}

We used Ngene V.1.2 software (Choice-Metrics, Sydney, Australia) to conduct the D-efficiency experimental design. After obtaining priors of the attributes and levels from the pilot, the Bayesian-efficient design was used to create the formal choice sets, which comprised 48 pairs of scenarios and were divided into six blocks, with eight pairs in each block. Blocking design boosted response efficiency by reducing the cognitive burden on respondents. $^{45}$

We applied unlabelled DCE, which had been widely used to investigate public preferences for healthcare. $^{16-18} 46-48$ Respondents in unlabelled DCEs found that they were not subject to the psychological cues of the labels, thus reflecting the real-life choice. ${ }^{49}{ }^{50}$ Also, in our research, we did not investigate patients' preferences for specific types of healthcare facilities. Therefore, the unlabelled DCE was considered appropriate. When no option had a definitive advantage, it was assumed that an opt-out option could raise the probability of neutral responses, increasing the number of individuals that might choose the opt-out scenario. ${ }^{51-53}$ While the forced-choice sets under preference uncertainty would favour options that were easier to justify and contributed to a lower likelihood of regret and error. ${ }^{54}$ Consequently, forced-choice sets were used in our DCE. Examples of choice scenarios were shown in online supplemental appendix 4 .

The questionnaire included four parts. The first part consisted of patients' socio-demographic characteristics, medical history, comorbidities and healthcare experience (ie, types of frequently visited healthcare facilities). The second part contained the DCE tasks. The third part was the items of EQ-5D-5L developed by the EuroQol Group. The EQ-5D-5L is a generic preference-based instrument that measures individuals' HRQoL. It used a health-state classification system defining health in five dimensions, mobility, self-care, usual activities, pain/discomfort and anxiety/depression. ${ }^{55}$ Each of the five dimensions was classified into five levels of perceived problems, no problem, mild problems, moderate problems, severe problems and unable to/extreme problems. The final part was the level of understanding and confidence when making the DCE choices. The score ranged from 0 (worst case) to 10 (best case) (online supplemental appendix 5). We excluded the DCE questionnaires with an average score of less than eight to ensure the validity of the data.

\section{Sample size}

There was no universal standard for the ideal sample size for DCEs ${ }^{56} \mathrm{~A}$ less efficient design might require a larger sample size, leading to increased costs. ${ }^{57}$ We followed a rule-of-thumb ${ }^{58}$ when determining sample size:

$$
\frac{n t a}{c} \geq 500
$$

where $\mathrm{n}$ was the number of respondents, $\mathrm{t}$ was the number of tasks, a was the number of alternatives, and $\mathrm{c}$ was the largest number of levels for any attributes. We had six blocks of choice sets; as a result, the minimum sample size was 564 .

\section{DCE implementation and data collection}

Our formal DCE was carried out from 1 November 2020 to 31 December 2020, in Jiangsu province and Shanghai municipality. Both Jiangsu province and Shanghai belong to the Yangtze River Delta region, which is the largest urban agglomeration in China. In recent years, the integration of healthcare resources and services in the region has been listed in the Chinese government's agenda. 
Inclusion criteria were patients aged 18 years or older, with a history of hypertension for at least 2 years, and who took medications regularly. Hypertension patients during pregnancy were excluded. Patients were recruited consecutively from nine healthcare facilities.

To ensure the validity and reliability of the survey, the DCE questionnaires were administrated through oneto-one, face-to-face interviews. Our interviewers consisted of eight medical interns and nine physicians. For quality assurance, we compiled a survey training manual and trained the interviewers before the experiment. The interviewers were required to check the completeness of each questionnaire immediately after it was completed. As long as any missing information, they had to ask patients to provide additional information. For patients who were illiterate or had blurred vision, the interviewers explained the meaning of the questionnaire item by item until the patients fully understood each item.

We proposed a hypothetical situation of poor blood pressure control and severely uncomfortable symptoms. Patients were asked to think carefully and make a trade-off between two types of services for their first-contact care. The duration of the survey ranged from $20 \mathrm{~min}$ to 1 hour. Patients were informed that participation in the survey was anonymous and voluntary, and their verbal and informed consent was obtained prior to the survey. We gave each patient a wrapped cotton towel as a gift (¥10 or US\$1.4).

\section{Patient and public involvement}

One hundred and eight patients participated in the pilot survey to provide feedback on the intelligibility and acceptability of the questionnaire. Responses from the patients contributed to a more apprehensible and concise description of the DCE questions. The patients engaged in the pilot were not involved in the formal survey. No patients took part in the recruitment of study participants or the carry out of the study.

\section{Statistical analysis}

Our DCE data analysis was based on the random-effects model. ${ }^{59}$ In the random utility theory, the conventional utility function $U$ consists of two parts: one is the determinism $V$ containing the observable component, and the other is the random component $\varepsilon$ representing the random error term with standard statistical properties. ${ }^{60}$ Therefore, the utility of the individual $i$ of alternative $n$ is:

$$
U_{\text {in }}=V_{\text {in }}+\varepsilon_{\text {in }}
$$

According to equation (1), the probability of the respondent choosing designated healthcare services was simulated. The probability of choice was determined by the indirect utility function of the respondent $i$ who choose $j$ in the selection set $s$. It was assumed to be a linear and additive form, and its form was:

$$
V_{i j s}=X_{i j s} \beta+\varepsilon_{i j s}
$$

Where $V_{i j s}$ represented the utility derived from a choice, $X_{i j s} \beta$ was the utility component, and $\varepsilon$ was the random component. The $X_{i j s} \beta$ was specified below, where $\beta_{1-6}$ represented reference scores of attributes and $\beta_{0}$ was the constant:

$$
\begin{aligned}
X_{i j s} \beta_{j}= & \beta_{0}+\beta_{1} \text { Treatment effects }_{j}{ }^{+} \\
& \beta_{2} \text { Physician }- \text { patient communication } j^{+} \\
& \beta_{3}{\text { Continuity of care } j^{+}}^{+} \\
& \beta_{4}{\text { Waiting } \text { time }_{j}+}^{+} \\
& \beta_{5} \text { Travel time }_{j}{ }^{+} \\
& \beta_{6} \text { Out }- \text { of }- \text { pocket costs }{ }_{j}
\end{aligned}
$$

We implemented the above equation by mixed logit regression using STATA V.14.2 SE (STATA) and was specified with 500 Halton draws. The mixed logit model allows for unknown heterogeneity in individual preferences. We assumed that all variables of the attributes had a random component and that the weights of preference were normally distributed. ${ }^{61}$ The choice of patients was the dependent variable, and the selected attributes were independent variables.

Respondents' characteristics are likely to influence their decisions, but they are neither part of the choice alternatives nor a direct source of utility. One way to investigate how respondents' characteristics affect their choices is to include interaction terms between attributes and individuals' characteristics, allowing weights of the attributes to vary with characteristics. ${ }^{62}$ Therefore, we extended the main effects model with interaction terms between attribute levels and the factors likely to influence patients' choices. The interaction terms were specified as random parameters to keep suitable computation times. To assess whether preferences varied, we performed $\chi^{2}$ tests for joint significance. SEs were clustered at the respondent level during the analysis.

Effects coding was used for categorical variables in DCE data. For effects coding, the mean effect for each attribute was normalised at zero, rather than all the reference categories being set to zero. ${ }^{63}$ Each coefficient was estimated relative to the mean attribute effect. ${ }^{63}$ The marginal rate of substitution between attributes could be obtained by calculating the ratio of the partial derivatives of each attribute, where $\beta$ was the coefficient of the attribute.

$$
M R S=-\frac{\beta_{a}}{\beta_{b}}
$$

Since our DCE attributes included costs, it could be used to generate an estimate of willingness to pay (WTP) of attributes expressed as in the unit of cost by replacing the denominator with the $\beta$ estimate for the cost attribute. According to the estimated preference scores for each attribute level, WTP for changing attribute A from level 1 to level 2 could be calculated as follows:

$$
\mathrm{WTP}=-\frac{\beta_{\mathrm{A} 2}-\beta_{A 1}}{\beta_{\text {cost }}}
$$


where $\beta_{\text {cost }}$ was the preference score of out-of-pocket costs, and $\beta_{A 1}$ and $\beta_{A 2}$ were preference scores of level 1 and level 2 for the attribute A, respectively.

\section{RESULTS}

\section{Patients' characteristics}

A total of 722 hypertension patients were consented to participate in our DCE survey. Nineteen patients were excluded from the analysis due to non-compliance with the inclusion criteria, incomplete data, lack of understanding and confidence in making the DCE choices. As a result, data from 703 patients were available for analysis. Two hundred and seven patients (29.45\%) were enrolled from primary healthcare facilities, 247 (35.13\%) from secondary hospitals and 249 (35.42\%) from tertiary hospitals. For details about the number of patients in each sampled hospital, please refer to online supplemental appendix 6 . On average, patients found it easy to understand the scenarios $(8.23,95 \% \mathrm{CI} 8.18$ to 8.27$)$, and confident in their choice $(8.99,95 \%$ CI 8.92 to 9.05$)$.

Table 2 summarises the sociodemographic and clinical characteristics of patients. The sample had more males than females $(56.90 \%$ vs $43.10 \%)$. The average age was 64.66years old (ranging from 24 to 96 years old). 38.26\% of the monthly household income was less than $¥ 4000$. A total of 416 patients $(59.17 \%)$ had comorbidities, and cardiovascular disease (191 patients) was the most common type (online supplemental appendix 7). Only $47.80 \%$ of patients considered primary healthcare facilities as their first choice, and only $26.17 \%$ of patients had contract service with general practitioners.

\section{Model estimation of preferences}

We found that patients valued healthcare services that generated good treatment effects $(\beta=4.502, \mathrm{p}<0.001)$, followed by travel time to healthcare facilities within 1 hour $(\beta=1.285, p<0.001)$, and the adequate physicianpatient communication $(\beta=0.771, \mathrm{p}<0.001)$ (table 3$)$. Minimal waiting time $(\beta=0.447, p<0.001)$ and continuity of care $(\beta=0.321, p<0.001)$ were also positive predictors of patients' choice of healthcare services. While out-ofpocket cost was a negative predictor of patients' preferences $(\beta=-0.168, \mathrm{p}<0.001)$. The SD revealed coefficient heterogeneity in the random parameters of attributes. We excluded patient data from healthcare facilities in Shanghai to do the sensitivity analysis, and the statistical significance of attributes was stable (online supplemental appendix 8).

\section{Marginal WTP}

According to the average WTP (table 4), we found that patients highly valued the magnitude of treatment effects. They would be WTP an extra ¥2489 for healthcare services to improve the effects from poor to good, while their WTP to increase the effects from poor to moderate was $¥ 1155$. The WTPs for other attributes from high to low were as follows: travel time, satisfied physician-patient

\begin{tabular}{|c|c|}
\hline Variables & $\mathbf{N}(\%)$ \\
\hline \multicolumn{2}{|l|}{ Gender } \\
\hline Male & $400(56.90)$ \\
\hline Female & $303(43.10)$ \\
\hline \multicolumn{2}{|l|}{ Age $^{*}$} \\
\hline$<65$ & $308(43.81)$ \\
\hline $65-74$ & $258(36.70)$ \\
\hline$\geq 75$ & $137(19.49)$ \\
\hline \multicolumn{2}{|l|}{ Education } \\
\hline Primary school/unschooled & $337(47.94)$ \\
\hline Junior high school/high school & 279 (39.69) \\
\hline Junior college or higher vocational college & $54(7.68)$ \\
\hline Bachelor's degree or above & $33(4.69)$ \\
\hline \multicolumn{2}{|l|}{ Employment } \\
\hline Farmer & $278(39.54)$ \\
\hline Urban employee & $106(15.08)$ \\
\hline Freelancers & $74(10.53)$ \\
\hline Unemployed & $22(3.13)$ \\
\hline Retiree & $223(31.72)$ \\
\hline \multicolumn{2}{|l|}{ Type of public health insurance } \\
\hline UEBMI & $272(38.69)$ \\
\hline URRBMI & $431(61.31)$ \\
\hline \multicolumn{2}{|l|}{ Monthly household income (CNY) } \\
\hline$\leq ¥ 2000$ & $126(17.92)$ \\
\hline$¥ 2001-¥ 4000$ & $143(20.34)$ \\
\hline$¥ 4001-¥ 6000$ & $130(18.49)$ \\
\hline$¥ 6001-¥ 8000$ & $91(12.95)$ \\
\hline$¥ 8001-¥ 10000$ & $72(10.24)$ \\
\hline$¥ 10001-¥ 12000$ & $54(7.68)$ \\
\hline$>¥ 12000$ & $87(12.38)$ \\
\hline \multicolumn{2}{|l|}{ Duration after diagnosis of hypertension (years) } \\
\hline$\leq 10$ & $474(67.43)$ \\
\hline$>10$ & $229(32.57)$ \\
\hline \multicolumn{2}{|l|}{ Comorbidities } \\
\hline No & $287(40.83)$ \\
\hline Yes & $416(59.17)$ \\
\hline \multicolumn{2}{|l|}{ The most frequently visited healthcare facilities } \\
\hline Primary healthcare facilities & $336(47.80)$ \\
\hline Secondary hospitals & $228(32.43)$ \\
\hline Tertiary hospitals & $139(19.77)$ \\
\hline \multicolumn{2}{|l|}{ Contract service with general practitioners } \\
\hline No & $519(73.83)$ \\
\hline Yes & $184(26.17)$ \\
\hline \multicolumn{2}{|l|}{ EQ-5D-5L index value $†$} \\
\hline$\leq 0.85$ & $423(60.17)$ \\
\hline$>0.85$ & $280(39.83)$ \\
\hline
\end{tabular}




\section{Table 2 Continued}

Variables N (\%)

*Patients were divided into three groups: young and middle-aged (younger than 65 years old), young-old elderly (aged 65-74), old-old elderly (aged 75 and older). ${ }^{76}$

†EQ-5D-5L represents the five-dimensional five-level instrument to measure health-related quality of life developed by the EuroQol Group. The utility index was derived from the Chinese value sets. ${ }^{77}$

CNY, Chinese yuan; UEBMI, Urban Employees Basic Medical Insurance; URRBMI, Urban-Rural Residents Basic Medical Insurance.

communication, minimum waiting time, moderate physician-patient communication, continuity of care, and moderate waiting time.

\section{Preference heterogeneity}

The impact of patients' characteristics on preferences for healthcare services was shown in online supplemental appendix 9. We tested for interactions of monthly household income levels with different attributes. Compared with low-income patients, those who had high income showed stronger preferences for good physician-patient communication $(\beta=0.377, \mathrm{p}<0.05)$ and minimum waiting

\begin{tabular}{|c|c|c|}
\hline Attributes & Mean (SE) & SD (SE) \\
\hline \multicolumn{3}{|l|}{ Treatment effects } \\
\hline Poor(ref) & $-4.299^{\star \star \star}(0.348)$ & \\
\hline Moderate & $-0.204^{*}(0.089)$ & $0.824^{\star * *}(0.160)$ \\
\hline Good & $4.502^{\star \star \star}(0.357)$ & $2.148^{\star \star \star}(0.223)$ \\
\hline \multicolumn{3}{|c|}{ Physician-patient communication } \\
\hline Poor(ref) & $-0.727^{\star \star \star}(0.089)$ & \\
\hline Moderate & $-0.044(0.061)$ & $-0.390^{* *}(0.147)$ \\
\hline Good & $0.771^{\star \star \star}(0.084)$ & $0.657^{\star \star \star}(0.119)$ \\
\hline \multicolumn{3}{|l|}{ Continuity of care } \\
\hline No(ref) & $-0.321^{\star \star \star}(0.048)$ & \\
\hline Yes & $0.321^{\star \star \star}(0.048)$ & $0.318^{* *}(0.121)$ \\
\hline \multicolumn{3}{|l|}{ Waiting time } \\
\hline \multicolumn{3}{|c|}{4 hours or longer (ref) $-0.476^{\star \star \star}(0.072)$} \\
\hline 2 hours & $0.029(0.063)$ & $-0.137(0.225)$ \\
\hline Within 0.5 hour & $0.447^{\star \star \star}(0.066)$ & $0.351^{\star \star}(0.132)$ \\
\hline \multicolumn{3}{|c|}{ Travel time } \\
\hline \multicolumn{3}{|c|}{6 hours or longer (ref) $-1.490^{\star \star \star}(0.122)$} \\
\hline 3 hours & $0.205^{\star \star \star}(0.061)$ & $0.409^{\star \star \star}(0.122)$ \\
\hline Within 1 hour & $1.285^{\star \star \star}(0.107)$ & $0.952^{\star \star \star}(0.111)$ \\
\hline \multicolumn{3}{|c|}{ Out-of-pocket costs (if reimbursed) } \\
\hline Cost (per ¥50) & $-0.168^{\star \star \star}(0.020)$ & $0.198^{\star * \star}(0.033)$ \\
\hline Log likelihood & -2299.4957 & \\
\hline Observations & 11248 & \\
\hline
\end{tabular}

The coefficient for the reference group was calculated as the negative sum of other coefficients. ${ }^{63}$

${ }^{*} \mathrm{P}<0.05 ;{ }^{* *} \mathrm{p}<0.01 ;{ }^{* * *} \mathrm{p}<0.001$.

HRQoL, Health-related quality of life; Ref, reference.;
Table 4 Marginal willingness to pay (WTP) for each attribute $(\mathrm{N}=703)$

\begin{tabular}{|c|c|}
\hline Attributes & WTP $(95 \%$ Cl) \\
\hline \multicolumn{2}{|l|}{ Treatment effects } \\
\hline From poor to moderate & $1155^{\star \star \star}(927$ to 1383$)$ \\
\hline From poor to good & $2489^{\star \star \star}$ (2013 to 2965$)$ \\
\hline \multicolumn{2}{|l|}{ Physician-patient communication } \\
\hline From poor to moderate & $191^{* \star *}(113$ to 270$)$ \\
\hline From poor to good & $423^{\star \star \star}(315$ to 532$)$ \\
\hline \multicolumn{2}{|l|}{ Continuity of care } \\
\hline From no to yes & $184^{\star * \star}(122$ to 247$)$ \\
\hline \multicolumn{2}{|l|}{ Waiting time } \\
\hline $\begin{array}{l}\text { From } 4 \text { hours or longer to } 0.5- \\
2 \text { hours }\end{array}$ & $146^{\star \star \star}$ (73 to 219$)$ \\
\hline $\begin{array}{l}\text { From } 4 \text { hours or longer to within } \\
0.5 \text { hour }\end{array}$ & $265^{\star \star \star}(185$ to 346$)$ \\
\hline \multicolumn{2}{|l|}{ Travel time } \\
\hline $\begin{array}{l}\text { From } 6 \text { hours or longer to } \\
1-3 \text { hours }\end{array}$ & $481^{\star \star \star}(368$ to 594$)$ \\
\hline $\begin{array}{l}\text { From } 6 \text { hours or longer to within } \\
1 \text { hour }\end{array}$ & $783^{\star \star \star}(615$ to 950$)$ \\
\hline
\end{tabular}

${ }^{\star \star *} \mathrm{P}<0.001$.

time $(\beta=0.396, p<0.01)$ (model 1$)$. The negative interaction term between income and moderate treatment effects showed that high-income patients valued the moderate effects to be less important than did low-income patients.

Similarly, we tested for interactions of age with the attributes, with young or mid-aged patients as the reference category (model 2). Four interaction terms were statistically significant: good treatment effects $(\beta=2.839$, $\mathrm{p}<0.001)$, shortest travel time $(\beta=0.533, \mathrm{p}<0.01)$, good physician-patient communication $(\beta=0.442, \mathrm{p}<0.05)$ and continuity of care $(\beta=0.232, p<0.05)$.

There were statistically significant interaction terms of comorbidities with three attributes. Patients who had comorbidities favoured more in healthcare services that generated good treatment effects $(\beta=0.986, p<0.05)$, required minimum travel time $(\beta=0.588, \mathrm{p}<0.01)$, and ensured continuity of care $(\beta=0.318, p<0.01)$ (model 3$)$.

Compared with patients who usually visited primary healthcare facilities, those who tended to seek healthcare services from secondary or tertiary hospitals expressed a stronger preference for good treatment effects $(\beta=0.898$, $\mathrm{p}<0.05)$ and minimum waiting time $(\beta=0.351, \mathrm{p}<0.05)$ (model 4). Patients with higher HRQoL paid more attention to healthcare services that contributed to good treatment effects $(\beta=1.748, \mathrm{p}<0.01)$ (model 5$)$.

\section{DISCUSSION}

Patients' preferences for healthcare services

To the best of our knowledge, this is the first DCE that systematically investigated the attributes influencing the 
choice of healthcare services for first-contact care among chronic disease patients like hypertension in China. An in-depth understanding of patients' perspectives on different healthcare service attributes is of global interest since it could inform the providing of appropriate healthcare that could improve patient satisfaction and service utilisation.

According to the estimated attribute-level coefficients, we found that the treatment effect was the most important attribute defining patients' preferences. Our results also showed that older adults, patients who had higher HRQoL, with comorbidities, and who usually visited secondary or tertiary hospitals to treat hypertension cared more about good treatment effects. The findings highlight the importance of taking effectiveness into account to improve patients' acceptance of primary healthcare services. However, the clinical experience of physicians ${ }^{1517}$ and types of healthcare professionals, ${ }^{16}$ rather than treatment effects, were often used to reflect the capabilities of healthcare provision in previous studies. In fact, preferences for provider types, which involved gender, types of medical staff, job titles, and professional training experience, were complex and difficult to interpret.

Our findings demonstrated that the minimum travel time to the healthcare facility was the second most important attribute. The results were consistent with previous DCEs, as the respondents disliked travelling longer distances to the healthcare providers. ${ }^{1644}$ Patients who were older and those who had comorbidities might feel inconvenient to travel a long distance for the firstcontact care and rated shortest travel time to be more important than the counterparts.

Continuity of care was concerned with the quality of care over time. In our study, continuity of care was defined as coordinated and patient-centred care. It is a process involving the orderly, uninterrupted movement of patients among the diverse elements of the service delivery system. ${ }^{43}$ We found that patients desired healthcare services that were consistent and coordinated according to their health needs. In addition, the continuity of care was considered even more important for older patients and patients who had comorbidities. They needed more health resources than other groups, and their choices of first-contact care should be paid more attention to.

We found that good physician-patient communication was also an important attribute preferred by patients. In fact, effective physician-patient communication is essential in healthcare, affecting the patients' compliance with recommendations for care. ${ }^{64}$ Physician-patient communication is a powerful indicator of healthcare quality that can determine patients' self-management behaviour and satisfaction with healthcare providers. ${ }^{65} 66$ This is shown by greater patient involvement and decisions are reached through shared decision making. ${ }^{67}$ Our analyses revealed that high income and older patients valued good physician-patient communication more than did lowerincome and younger groups.
Longer waiting time and increased out-of-pocket costs were significant, negative predictors for the entire sample, showing that patient preferences decreased as the waiting time and out-of-pocket costs increased. Similar results were noted in DCEs eliciting public preferences for the choice of healthcare providers. ${ }^{4750}$ In addition, we found high-income patients and those who were likely to visit secondary or tertiary hospitals concerned more about waiting time. As shown in the previous study conducted in a Chinese public tertiary hospital, the reduced waiting time led to increased patient satisfaction. ${ }^{68}$ Patients might have an increased WTP for services that require less waiting time for an appointment to diagnose or treat, especially in the case of severe symptoms. ${ }^{69}$

\section{Implications of the study findings}

In China, patients sought first-contact care in a disorderly manner, and the gate-keeping role of primary healthcare has not been fully implemented. ${ }^{70}$ Patients' preferences should be known to guide the delivery of appropriate, effective and efficient care. Our research confirms that the ideal healthcare services that meet hypertension patients' demands for first-contact care comprise the following attributes: produce good treatment effects, closer to home, offer good physician-patient communication, need short waiting time, ensure continuity of care and require low out-of-pocket costs per visit.

The treatment effect was the most important attribute to attract patients. Patients were more likely to choose the healthcare services that led to the experience of good quality. It is worth noting that older patients, patients who had high HRQoL, patients with comorbidities, and those who tended to visit secondary or tertiary hospitals would pay special attention to treatment effects. Meanwhile, patients expect to communicate with physicians to deliberate and express their preferences and views during the clinical decision-making process. Furthermore, older patients who are emotionally vulnerable and socially isolated are particularly in need of the emotional, social and practical support that sensitive physician-patient communication can provide. ${ }^{71}$

Evidence of variations in the perceived utility of healthcare services among patients emphasises the importance of taking individual patient preferences into account to address the problems of inadequate primary healthcare service utilisation and the ineffectiveness of the twoway referral mechanism. Healthcare systems need to be adaptable enough to offer patients choices to account for heterogeneity in patients' preferences.

Our study highlights the importance of improved service quality, timely access, and shared decision-making for the first-contact care of chronic diseases like hypertension. The improvement of service quality, physicianpatient communication, and continuity of care will contribute to patients' preference to choose primary healthcare facilities as the first-contact care. Our findings were consistent with the optimal healthcare delivery strategies to achieve universal health coverage, which involves 
providing effective, safe, people-centred care that is timely, equitable, integrated and efficient. ${ }^{72}$

The quality of primary healthcare in China needed to be strengthened, and evidence-based monitoring and evaluation of the service quality are crucial for attaining the goals of healthcare system reform. ${ }^{70}$ In addition to education for the general practitioners, the Chinese government could consider tailoring continuing training for the primary healthcare workforce ${ }^{73}$ Shared decision making is appropriate for clinical decisions involving multiple reasonable options, ${ }^{74}$ such as the management of chronic diseases. To improve the physicians' communication skills, strictly planned, culturally competent, effectively implemented and rigorously evaluated trainings are required. ${ }^{75}$ Care coordination approaches should also be advocated to engage patients in decision making, support effective management of comorbidities, and ensure accessibility to interventions. Furthermore, the primary healthcare-based integrated delivery system in China should be strengthened. ${ }^{13}$

\section{Strengths and limitations}

Our DCE provides valuable information about how patients weigh their first-contact care options and trade-off different healthcare service features. A better understanding of patients' preferences will guide the future development of the two-way referral mechanism, as policy-makers aim to bridge the gap between the optimal modes for patient-centred service delivery and patients' first-contact care needs.

The major contributions of our study are as follows. First, we used a DCE which followed good research practices, offering the advantage to explore the trade-offs between attributes of healthcare services. Second, the Bayesian-efficient design was applied to increase the statistical efficiency of the choice sets design, and a blocking technique was used to increase the response efficiency of patients. Third, we derived WTP estimates in hypothetical settings among patients with chronic diseases like hypertension. Fourth, this is the most comprehensive study that identifies preference heterogeneity according to age, income, HRQoL, comorbidities and past healthcare service experience.

Our study has several limitations. First, the DCE results are not representative of all patients with chronic diseases, because we only explored the preferences among hypertension patients to ensure the homogeneity of patients. Future studies need to enrol patients with other types of chronic diseases and identify variations in patients' preferences across different subgroups. Second, our samples were from Jiangsu and Shanghai, which stand for the most economically developed regions in China. Future studies should have a nationally representative sample by including the economically underdeveloped regions. Meanwhile, evenly distribution of sampled healthcare facilities in each region should be ensured. Third, given the limited number of attributes and levels tested in DCE, it might not represent complex real-life situations.
To further understand the relationship between stated (those elicited in the DCE) and revealed preferences (actual first-contact care-seeking behaviour), studies are warranted to investigate if and how patients' preferences in healthcare services impact their long-term clinical outcomes. Finally, we only used comorbidity to represent disease progression and severity. Researches are suggested to evaluate variations of patients' preferences at different stages of the disease.

\section{CONCLUSION}

Our DCE provides evidence about how hypertension patients value the attributes of healthcare services, including the capabilities, efficiency, affordability and convenience of service provision, in the context of chaotic first-contact care-seeking behaviour in China. The findings underline the importance of effective, convenient, efficient, coordinated and patient-centred care for chronic diseases like hypertension. We also found preference heterogeneity that is correlated with patients' sociodemographic characteristics, feelings of health conditions, the severity of disease (ie, comorbidities) and the prior experience of healthcare services. Policy-makers and healthcare providers are suggested to work on aligning the service provision with patients' preferences, thus promoting the rational utilisation of healthcare resources.

Acknowledgements We acknowledge the contributions made by our interviewers who did one-to-one, face-to-face interviews with the patients. We are grateful to the patients for their time and efforts. We sincerely thank the reviewers for providing valuable comments and suggestions on the manuscript. We acknowledge the guidance from Professor Hao Yu from Harvard Medical School and Harvard Pilgrim Healthcare Institute on the research design. At the time of drafting the research protocol, Jinsong Geng was a fellow at the Fellowship in Health Policy and Insurance Research, Department of Population Medicine, Harvard Medical School and Harvard Pilgrim Healthcare Institute.

Contributors JG, $\mathrm{XY}$ and $\mathrm{HB}$ led the design and analysis of the discrete choice experiment. JS and $X Y$ contributed to the literature search and data interpretation. $X Y, L Q$ and ZF contributed to implementing the discrete choice experiment. XY, $\mathrm{HB}$ and $\mathrm{JG}$ performed the statistical analysis and wrote the manuscript. JG is the guarantor for the study. JG accepts full responsibility for the finished work and/ or the conduct of the study, had access to the data, and controlled the decision to publish

Funding This work was supported by the MOE (Ministry of Education in China) Project of Humanities and Social Sciences (Grant No. 21YJAZH023), Science and Technology Project of Nantong City (Grant No. MS12021064), National Natural Science Foundation of China (Grant No. 71603138), Nantong Municipal Health Commission (Grant No. 2020JCC003) and Key Project of Technology Innovation Think Tanks in Nantong (Grant No. CXZK202002). The funders provided financial support for the conduct of the study.

Disclaimer The funders had no role in the design, implementation, data collection and statistical analysis, data interpretation, or writing of the manuscript.

Competing interests None declared.

Patient consent for publication Not applicable.

Ethics approval This study, including the patient consent process, has been approved by the Medical Ethics Committee in Nantong University (Ethical Approval-202054) and conforms to the ethical guidelines of the Declaration of Helsinki.

Provenance and peer review Not commissioned; externally peer reviewed. 
Data availability statement Data are available on reasonable request. Data will be available on reasonable request to the corresponding author.

Supplemental material This content has been supplied by the author(s). It has not been vetted by BMJ Publishing Group Limited (BMJ) and may not have been peer-reviewed. Any opinions or recommendations discussed are solely those of the author(s) and are not endorsed by BMJ. BMJ disclaims all liability and responsibility arising from any reliance placed on the content. Where the content includes any translated material, BMJ does not warrant the accuracy and reliability of the translations (including but not limited to local regulations, clinical guidelines, terminology, drug names and drug dosages), and is not responsible for any error and/or omissions arising from translation and adaptation or otherwise.

Open access This is an open access article distributed in accordance with the Creative Commons Attribution Non Commercial (CC BY-NC 4.0) license, which permits others to distribute, remix, adapt, build upon this work non-commercially, and license their derivative works on different terms, provided the original work is properly cited, appropriate credit is given, any changes made indicated, and the use is non-commercial. See: http://creativecommons.org/licenses/by-nc/4.0/.

\section{ORCID iDs}

Jianwei Shi http://orcid.org/0000-0002-3523-6246

Jinsong Geng http://orcid.org/0000-0003-3389-9051

\section{REFERENCES}

1 Yusuf S, Joseph P, Rangarajan S, et al. Modifiable risk factors, cardiovascular disease, and mortality in 155722 individuals from 21 high-income, middle-income, and low-income countries (pure): a prospective cohort study. Lancet 2020;395:795-808.

2 National Health Commission of the People's Republic of China. China health statistics Yearbook. Beijing: National Health Commission of the People's Republic of China, 2020: 239-40.

3 Mahajan S, Feng F, Hu S, et al. Assessment of prevalence, awareness, and characteristics of isolated systolic hypertension among younger and middle-aged adults in China. JAMA Netw Open 2020;3:e209743.

4 Wang Z, Chen Z, Zhang L, et al. Status of hypertension in China: results from the China hypertension survey, 2012-2015. Circulation 2018;137:2344-56.

5 Lu J, Lu Y, Wang X, et al. Prevalence, awareness, treatment, and control of hypertension in China: data from 1.7 million adults in a population-based screening study (China peace million persons project). Lancet 2017;390:2549-58.

6 Wong MCS, Wang HHX, Cheung CSK, et al. Factors associated with multimorbidity and its link with poor blood pressure control among 223,286 hypertensive patients. Int J Cardiol 2014;177:202-8.

$7 \mathrm{Gu} \mathrm{S}$, Hu H, Dong H. Systematic review of the economic burden of pulmonary arterial hypertension. Pharmacoeconomics 2016;34:533-50.

8 Tao W, Zeng Z, Dang H, et al. Towards universal health coverage: lessons from 10 years of healthcare reform in China. BMJ Glob Health 2020;5:e002086.

9 Li M, Zhang Y, Lu Y, et al. Factors influencing two-way referral between hospitals and the community in China: a system dynamics simulation model. Simulation 2018;94:765-82.

$10 \mathrm{Ta}$ Y, Zhu Y, Fu H. Trends in access to health services, financial protection and satisfaction between 2010 and 2016: has China achieved the goals of its health system reform? Soc Sci Med 2020;245:112715.

11 Zhang A, Nikoloski Z, Albala SA, et al. Patient choice of health care providers in China: primary care facilities versus hospitals. Health Syst Reform 2020;6:e1846844.

12 Sun J, Hu G, Ma J, et al. Consumer satisfaction with tertiary healthcare in China: findings from the 2015 China national patient survey. Int J Qual Health Care 2017;29:213-21.

13 Yip W, Fu H, Chen AT, et al. 10 years of health-care reform in China: progress and gaps in universal health coverage. Lancet 2019;394:1192-204.

14 Wu D, Lam TP, Lam KF, et al. Health reforms in China: the public's choices for first-contact care in urban areas. Fam Pract 2017;34:194-200.

15 Miners AH, Llewellyn CD, Cooper VL, et al. A discrete choice experiment to assess people living with HIV's (PLWHIV's) preferences for GP or HIV clinic appointments. Sex Transm Infect 2017;93:105-11.

16 Wong SF, Norman R, Dunning TL, et al. A discrete choice experiment to examine the preferences of patients with cancer and their willingness to pay for different types of health care appointments. $J$ Natl Compr Canc Netw 2016;14:311-9.

17 O'Hara NN, Slobogean GP, Mohammadi T, et al. Are patients willing to pay for total shoulder arthroplasty? Evidence from a discrete choice experiment. Can J Surg 2016;59:107-12.

18 Zanolini A, Sikombe K, Sikazwe I, et al. Understanding preferences for HIV care and treatment in Zambia: evidence from a discrete choice experiment among patients who have been lost to follow-up. PLoS Med 2018;15:e1002636.

19 Zhu J, Li J, Zhang Z, et al. Patients' choice and preference for common disease diagnosis and diabetes care: a discrete choice experiment. Int J Health Plann Manage 2019;34:e1544-55.

20 Dolan P, Tsuchiya A. Health priorities and public preferences: the relative importance of past health experience and future health prospects. J Health Econ 2005;24:703-14.

21 Garland SN, Eriksen W, Song S, et al. Factors that shape preference for acupuncture or cognitive behavioral therapy for the treatment of insomnia in cancer patients. Support Care Cancer 2018;26:2407-15.

22 van Oostveen CJ, Gouma DJ, Bakker PJ, et al. Quantifying the demand for hospital care services: a time and motion study. BMC Health Serv Res 2015;15:15:15.

23 Wellay T, Gebreslassie M, Mesele M, et al. Demand for health care service and associated factors among patients in the community of Tsegedie district, Northern Ethiopia. BMC Health Serv Res 2018;18:697.

24 World Health Organization. How to conduct a discrete choice experiment for health workforce recruitment and retention in remote and rural areas: a user guide with case studies. Geneva, 2012: 1-94.

25 Fletcher B, Hinton L, McManus R, et al. Patient preferences for management of high blood pressure in the UK: a discrete choice experiment. Br J Gen Pract 2019;69:e629-37.

26 Institute of Medicine Committee (US) on Quality of Health Care in America. Crossing the quality chasm: a new health system for the 21st century. Washington, DC: National Academies Press, 2001.

27 Jahanmehr N, Rashidian A, Khosravi A, et al. A conceptual framework for evaluation of public health and primary care system performance in Iran. Glob J Health Sci 2015;7:341-57.

28 Donabedian A. Evaluating the quality of medical care. 1966. Milbank Q 2005;83:691-729.

29 Fineberg HV. Shattuck Lecture. A successful and sustainable health system--how to get there from here. N Engl J Med 2012;366:1020-7.

30 Hua Q, Fan L, Li J. 2019 Chinese guideline for the management of hypertension in the elderly. J Geriatr Cardiol 2019;16:67-99.

31 Joint Committee for Guideline Revision. 2018 Chinese guidelines for prevention and treatment of Hypertension-A report of the revision Committee of Chinese guidelines for prevention and treatment of hypertension. J Geriatr Cardiol 2019;16:182-241.

32 World Health Organization. Guideline for the pharmacological treatment of hypertension in adults. Geneva: World Health Organization, 2021: 5-6.

33 National Institute for Health and Care Excellence. Hypertension in adults: diagnosis and management. London: National Institute for Health and Care Excellence, 2019.

34 The National Essential Public Health Service Program Office for Management of Hypertension in Primary Health Care, National Center for Cardiovascular Diseases, National Committee on Hypertension Management in Primary Health Care.. National clinical practice guidelines on the management of hypertension in primary health care in China. Chinese $J$ of Fron of Medi Sci 2021;13:26-37.

35 World Health Organization. Continuity and coordination of care: a practice brief to support implementation of the WHO framework on integrated people-centred health services. Geneva: World Health Organization, 2018: 9-22.

36 Health Quality Ontario. Continuity of care to optimize chronic disease management in the community setting: an evidence-based analysis. Ont Health Technol Assess Ser 2013;13:1-41.

$37 \mathrm{Ha}$ JF, Longnecker N. Doctor-patient communication: a review. Ochsner J 2010;10:38-43.

38 Ratcliffe J, Van Haselen R, Buxton M, et al. Assessing patients' preferences for characteristics associated with homeopathic and conventional treatment of asthma: a conjoint analysis study. Thorax 2002;57:503-8.

39 Tinelli M, Petrou P, Samoutis G, et al. Improving quality care for diabetes in the community: what do Cypriot patients want? Int J Qual Health Care 2018;30:443-9.

40 Whitty JA, Stewart S, Carrington MJ, et al. Patient preferences and willingness-to-pay for a home or clinic based program of chronic heart failure management: findings from the which? trial. PLoS One 2013;8:e58347

41 Albada A, Triemstra M. Patients' priorities for ambulatory hospita care centres. A survey and discrete choice experiment among 
elderly and chronically ill patients of a Dutch Hospital. Health Expect 2009;12:92-105

42 Ryan M, Bate A, Eastmond CJ, et al. Use of discrete choice experiments to elicit preferences. Qual Health Care 2001;10(Suppl 1):i55-60.

43 Gulliford M, Naithani S, Morgan M. What is 'continuity of care'? J Health Serv Res Policy 2006;11:248-50.

44 Wang X, Song K, Zhu P, et al. How do type 2 diabetes patients value urban integrated primary care in China? results of a discrete choice experiment. Int J Environ Res Public Health 2019;17:117.

45 Reed Johnson F, Lancsar E, Marshall D, et al. Constructing experimental designs for discrete-choice experiments: report of the ISPOR conjoint analysis experimental design good research practices Task force. Value Health 2013;16:3-13.

46 Beam NK, Bekele Dadi G, Rankin SH, et al. A discrete choice experiment to determine facility-based delivery services desired by women and men in rural Ethiopia. BMJ Open 2018;8:e016853.

47 Liu Y, Kong Q, de Bekker-Grob EW. Public preferences for health care facilities in rural China: a discrete choice experiment. Soc Sci Med 2019;237:112396.

48 Bahrampour M, Bahrampour A, Amiresmaili M, et al. Hospital service quality - patient preferences - a discrete choice experiment. Int $J$ Health Care Qual Assur 2018;31:676-83.

49 Kløjgaard ME, Bech M, Søgaard R. Designing a stated choice experiment: the value of a qualitative process. $J$ Choice Model 2012;5:1-18.

50 Zhu J, Li J, Zhang Z, et al. Exploring determinants of health provider choice and heterogeneity in preference among outpatients in Beijing: a labelled discrete choice experiment. BMJ Open 2019;9:e023363.

51 Seghieri C, Mengoni A, Nuti S. Applying discrete choice modelling in a priority setting: an investigation of public preferences for primary care models. Eur J Health Econ 2014;15:773-85.

52 Ryan M, Skåtun D. Modelling non-demanders in choice experiments. Health Econ 2004;13:397-402.

53 Veldwijk J, Lambooij MS, de Bekker-Grob EW, et al. The effect of including an opt-out option in discrete choice experiments. PLOS One 2014;9:e111805.

54 Dhar R, Simonson I. The effect of forced choice on choice. J Mark Res 2003;40:146-60.

55 Lu Y, Wang N, Chen Y, et al. Health-related quality of life in type-2 diabetes patients: a cross-sectional study in East China. BMC Endocr Disord 2017;17:1-7.

56 de Bekker-Grob EW, Donkers B, Jonker MF, et al. Sample size requirements for discrete- choice experiments in healthcare: a practical guide. Patient 2015;8:373-84.

57 Vanniyasingam T, Cunningham CE, Foster G, et al. Simulation study to determine the impact of different design features on design efficiency in discrete choice experiments. BMJ Open 2016;6:e011985.

58 Johnson R, Orme B. Sample size issues for conjoint analysis. In: Orme B, ed. Getting started with conjoint analysis: strategies for product design and pricing research. Madison: Research Publishers, 2010: 57-66.

59 Manski CF. The structure of random utility models. Theory Decis 1977;8:229-54

60 Nieboer AP, Koolman X, Stolk EA. Preferences for long-term care services: willingness to pay estimates derived from a discrete choice experiment. Soc Sci Med 2010;70:1317-25.
61 Phillips EA, Himmler SF, Schreyögg J. Preferences for e-mental health interventions in Germany: a discrete choice experiment. Value Health 2021;24:421-30.

62 Umar N, Quaife M, Exley J, et al. Toward improving respectful maternity care: a discrete choice experiment with rural women in northeast Nigeria. BMJ Glob Health 2020;5:e002135.

63 Hauber AB, González JM, Groothuis-Oudshoorn CGM, et al. Statistical methods for the analysis of discrete choice experiments: a report of the ISPOR conjoint analysis good research practices Task force. Value Health 2016;19:300-15.

64 Roberts MJ, Campbell JL, Abel GA, et al. Understanding high and low patient experience scores in primary care: analysis of patients' survey data for general practices and individual doctors. BMJ 2014;349:96034.

65 Matusitz J, Spear J. Effective doctor-patient communication: an updated examination. Soc Work Public Health 2014;29:252-66.

66 Alfred M, Ubogaya K, Chen X, et al. Effectiveness of culturally focused interventions in increasing the satisfaction of hospitalized Asian patients: a systematic review. JBI Database System Rev Implement Rep 2016;14:219-56.

67 Birkeland S, Bismark M, Barry MJ, et al. Is greater patient involvement associated with higher satisfaction? experimental evidence from a vignette survey. BMJ Qual Saf 2021. doi:10.1136/ bmjqs-2020-012786. [Epub ahead of print: 22 Apr 2021].

68 Sun J, Lin Q, Zhao P, et al. Reducing waiting time and raising outpatient satisfaction in a Chinese public tertiary General hospital-an interrupted time series study. BMC Public Health 2017; $17: 668$

69 Roll K, Stargardt T, Schreyögg J. Effect of type of insurance and income on waiting time for outpatient care. Geneva Pap Risk Insur Issues Pract 2012;37:609-32.

70 Liao R, Liu Y, Peng S, et al. Factors affecting health care users' first contact with primary health care facilities in North eastern China, 2008-2018. BMJ Glob Health 2021;6.

71 Williams SL, Haskard KB, DiMatteo MR. The therapeutic effects of the physician-older patient relationship: effective communication with vulnerable older patients. Clin Interv Aging 2007;2:453-67.

72 World Health Organization, International Bank for Reconstruction and Development, Organization for Economic Cooperation and Development. Delivering quality health services: a global imperative for universal health coverage. Geneva: World Health Organization, 2018: 27--39.

$73 \mathrm{Li} \mathrm{X,} \mathrm{Krumholz} \mathrm{HM,} \mathrm{Yip} \mathrm{W,} \mathrm{et} \mathrm{al.} \mathrm{Quality} \mathrm{of} \mathrm{primary} \mathrm{health} \mathrm{care} \mathrm{in}$ China: challenges and recommendations. Lancet 2020;395:1802-12.

74 Backman WD, Levine SA, Wenger NK, et al. Shared decisionmaking for older adults with cardiovascular disease. Clin Cardiol 2020;43:196-204.

75 Liu X, Rohrer W, Luo A, et al. Doctor-patient communication skills training in mainland China: a systematic review of the literature. Patient Educ Couns 2015;98:3-14.

76 Zhang P, Xiong J, Zeng J, et al. Clinical evaluation of active tuberculosis-related deaths in Shenzhen, China: a descriptive study. Int J Gen Med 2021;14:237-42.

77 Liang Z, Zhang T, Lin T, et al. Health-related quality of life among rural men and women with hypertension: assessment by the EQ-5D5L in Jiangsu, China. Qual Life Res 2019;28:2069-80. 